\title{
Intuitive Sensemaking: From Theory to Simulation Based Training
}

\author{
Kathleen Bartlett, Margaret Nolan, and Andrea Marraffino \\ MESH Solutions, LLC - A DSCI Company, Orlando, FL, USA \\ \{kbartlett, mnolan, amarraffino\} @mesh.dsci.com
}

\begin{abstract}
The concept of sensemaking has become a prominent component of military operations in ambiguous environments. Sensemaking, in general, describes the process of pattern recognition, semantic formulation, anticipation, and holistic understanding and supports sociocultural situation assessment, anomaly detection, and anticipatory thinking. This skill enables intuitive experts to rapidly draw accurate conclusions based on cues that others cannot discern or to attend to the most important cues, based on experience. Simulation-based training can enhance and accelerate the ability to recognize and analyze cues and patterns by translating the unconscious, automatic monitoring and integration practiced by experts into a conscious cognitive process that we call intuitive sensemaking. We describe an Office of Naval Research project, currently in development, intended to effectively train previously ambiguous advanced cognitive skills such as intuition-informed sensemaking. With training, teams of military personnel should see increases in cohesiveness, sociocultural situation assessment, anomaly detection, and anticipatory thinking.
\end{abstract}

Keywords: Sensemaking, Intuition, Simulation-Based Training, HumanComputer Interaction (HCI), Expertise, Implicit Learning.

\section{Introduction: Sensemaking and Intuition}

Sensemaking describes the ability to explain data that are sparse, noisy, and uncertain (Moore, 2011) when assessing a situation. Sociologist Karl Weick, one of the first academics to define sensemaking as it relates directly to complex operational environments, contended that the ability to construct a coherent and shared explanation for events and circumstances enables operational functioning during periods of great uncertainty (Weick, 1993). In the past, this type of intelligence gathering generally consisted of locating a known entity or a specific target; today's battlefields, however, require an additional skill set: looking for (and judging the significance of) undefined activities or transactions. Frequently, observers must scan complex, ambiguous settings and groups of diverse, unpredictable people to assess threats and determine necessary actions. They must make sense of situations that include large numbers of relatively small actors responding to a shifting set of situational factors (Moore, 2011). Predicting and anticipating the actions of these players and the directional 
shifts of surrounding circumstances requires enhanced observational and sensemaking training that accelerates the acquisition of expertise and fosters the development of intuition.

Individuals engage in sensemaking under conditions of equivocality and uncertainty (Weick, 1979, 1993), and their expectations and motivations affect this process, since individuals vary in how they construct ethical issues and make intuitive judgments about those constructions (Sonenshein, 2007). While some people seem to be more naturally intuitive than others, recent work suggests that the process of intuition rests on an unconscious awareness, valuation, and integration of cues that shape decisions and judgments, and in experts, those perceptual observations may reach a level of automaticity (e.g., Dervin, B., 1983; Klein, Moon, \& Hoffman, 2006b; Thurlow \& Mills, 2009; Betsch, 2008; Dane \& Pratt, 2007). When people "know" without knowing how they know, their conscious awareness may have been influenced by an unconscious monitoring of patterns and anomalies (e.g., Claxton, 2000; Simons \& Chabris, 2010). For example, profound decisions and actions that save firefighters in potentially catastrophic situations can most likely be credited to implicit processing of important environmental cues. Decades of research on implicit learning have shown that our brains possess an array of mechanisms for automatically extracting information from the environment without our awareness (Reber, 2008). This skill enables intuitive experts to rapidly draw accurate conclusions based on cues that others cannot discern or to attend to the most important cues, given conditions and context of a situation, based on experience.

The human brain has two distinct information processing systems: one conscious and deliberative and the other unconscious and intuitive. Intuition is rooted in the unconscious information processing system, as are related inputs of implicit attitudes and goals (Hassin, Uleman, \& Bargh, 2005). This intuitive processing creates the moment of intuition, the experience of knowing, without knowing how that knowledge came to be. Dane and Pratt (2007) offer this definition: intuitions are "affectively charged judgments that arise through rapid, non-conscious, and holistic associations" (Dane \& Pratt, 2007). Betsch (2008) provides a definition of the three core components of intuition: "Intuition is a process of thinking. The input to this process is mostly provided by knowledge stored in long-term memory that has been primarily acquired via associative learning. The input is processed automatically and without conscious awareness. The output of the process is a feeling that can serve as a basis for judgments and decisions." Thus, while experts may attribute their advanced awareness (e.g., sense of danger before an explosion) to intuition, at an unconscious level, they most likely had mentally observed, analyzed, and decided how to act without recognizing that cognitive process. Intuition typically emerges with no awareness of the mental events leading to it, which fits with our conjecture that implicit memory is critical in producing trustworthy intuition. The results of this implicit learning often appear as an intuition or a "sixth sense" about the current situation. Development of expertise that achieves this level of automaticity takes time, but simulation-based training can accelerate that process. 


\section{$2 \quad$ Can Sensemaking and Intuition Be Trained?}

Research suggests that fast, affect-rich intuitions frequently drive individuals' behavior (Loewenstein, 1996). Subject-matter experts routinely use their intuitive abilities to help them make decisions and judgments (Hodgkinson et al., 2009), and intuition is "critical to effective decision making in many settings" (Salas et al., 2009, p. 2). Emerging theory further proposes the existence of expertise-based intuition, a form of intuition rooted in domain-specific expertise that experts can learn to constructively employ in support of their decision making, sensemaking, and other cognitive processes (Salas et al., 2009). This suggests that intuition, like other cognitive mechanisms, can improve through experience, deliberate practice, and a variety of specialized training interventions. It also means that it may be possible to decrease the time required to effectively use intuition to drive decisions in situations where one has not had the requisite time required to become a domain expert. In other words, we may be able to artificially enhance intuitive decision making skills at a more rapid pace than previous research has suggested (Eriksson, 1996) by using specific training techniques within implicit learning environments.

Given the influence of intuition on cognitive performance, and considering the recent evidence that individuals can intentionally improve their intuitive skills, the military community's interest in intuition has grown. Because intuition and psychosocial skills complement one another, training for intuitive processing might be conveyed in the learning context of sociocultural pattern recognition, anticipation, and interaction. In that context, intuitive processing can enhance the discernment and interpretation of subtle sociocultural cues and patterns, and supports the need to enhance military personnel's sociocultural abilities. Moreover, such blended instruction on these topics may help engender the generalizable sociocultural competencies that Marines and Sailors need to excel in any operational environment.

Despite its subconscious facets, training and education can enhance individuals' intuitive capacities (Salas et al., 2009). Classically, experts build their intuitive skills through experience and implicit learning (Agor, 1989; Harper, 1989; Klein, 1998). They learn to regulate their intuitive feelings by actively seeking feedback (Hogarth, 2001), and they selectively attend to intuitive thoughts based upon the characteristics of the problem space (Salas et al., 2009). Fortunately, accelerated acquisition of domain experience and the development of intuition-related skills can be facilitated through deliberate practice, critical self-appraisal, and candid feedback (Hodgkinson, 2009). A validated training program for intuition could help military personnel improve their access to, and appropriate use of, intuition. For example, through implicit learning, situated training, deliberate practice, self-critique, and metacognitive instruction, warfighters could enhance their intuition-informed pattern recognition capabilities, learn to more rapidly and efficaciously conduct intuition-informed situation assessments, and gain regulatory skills to more deliberately control their intuitive processing.

Specifically, simulation-based training can enhance and accelerate the ability to recognize and analyze cues and patterns by translating the unconscious, automatic 
monitoring and integration practiced by experts into a conscious cognitive process that we call intuitive sensemaking. However, the challenge consists of taking empirically-driven findings about intuition, based on models and theories, best practices in simulation-based training, and what is known about instructional strategies and feedback, to design effective scenarios that will stimulate and train the development of these unconscious intuitive functions.

For simulation-based training, the scenario design provides the context for the training; it defines the capabilities of the simulation system that should be utilized to create the required conditions and cues, and suggests the instructional strategies that provide the best method to deliver the training and the performance feedback. Will it be simple or complex? Is a part-task trainer that adds layers as expertise improves the best option to train the objectives? Is immediate feedback or an after-action review the best way to emphasize learning points? Should feedback be embedded in the scenario design or mediated by a live instructor? Design decisions will also involve "visual noise" and temporal markers, as well as spatial issues of proximity, juxtaposition, and foreshadowing. How can we design a richly cued scenario that will enable or direct a search, and what are the salient cues important to the overall training objective that will need to be detected?

Event-based training featuring novel, unexpected situations offers friction points to stimulate decision making under time or mission constraints and to provide triggers for other courses of action; timely feedback can direct attention to cues and patterns that are missed. Simulations offer a chance to rehearse actions and thinking, but capturing the thinking and knowing that occurs below conscious awareness presents another challenge. Simulations also provide a means for repetition of the same or similar scenarios, increasing the complexity in a chained strategy, and provide the means for transfer of training to a novel scenario. Performance measurement is generally comprised of observable outcomes and courses of action taken based on decision(s) made in support of an objective. Simulation-based technologies can provide capture of voice and video recordings, resources and assets used, and digital information exchanged to help build metrics. Measurement of physiological functions (e.g., via EEG, eye tracking) provide data that may help identify what is being observed and when, for how long, in what sequence, and how that information influences decision making and outcomes. Correlating those data with trainee verbal protocol and demonstrable results from actions taken can provide insights into implicit decision making and how best to employ training feedback to uncover the trainees' strategies, such as how or if they took into account cue characteristics, multiple criteria, sequence of visual acquisition of cues, etc.

Scenario-based simulation training to accelerate the process of automaticity will need to develop the skills of an expert via intense and intentional practice with specific feedback that impacts the learner in an emotional, highly connotative way. Feedback delivery options must also be explored, since allowing learners to make errors can increase problem solving abilities and enhance the emotional impact of the experience, whereas providing delayed feedback, or after-action review, may create a disconnect between action and consequence (or may lead to negative training). Consensus among 
experts on what constitutes "good" decisions, and therefore actions, will need to be addressed to ensure consistent training objectives and sound scenario and metric design. Decomposition of the decisions and "micro" decisions that could or should be made within the context of a given scenario is paramount for simulation design.

\subsection{PercepTS: Immersive Technologies to Enhance Intuitive Decision Making}

We theorize that, after exploring factors and options related to simulation-based training, military researchers will have the ability to effectively train previously ambiguous advanced cognitive skills such as intuition-informed sensemaking. With training, teams of military personnel should see increases in cohesiveness, sociocultural situation assessment, anomaly detection, and anticipatory thinking.

Toward this end, the Office of Naval Research (ONR) stood up the Perceptual Training Systems and Tools (PercepTS) program to explore immersive methodologies and technologies for improving the training of sensemaking/perceptual knowledge, skills, and abilities (KSAs) in operational environments. This work includes development of an approach to enhance the decision-making skills of military personnel by investigating a range of cognitive training approaches, situated in the context of urban sociocultural sensemaking.

On-going PercepTS work seeks to develop an actionable framework of perceptual competencies and training strategies for military use. Based on this framework and the instructional strategies identified therein, a perceptual skils Program of Instruction (POI) suitable for implementation by military instructors could be developed under future projects, tested, and packaged as advanced instructional strategies for future use in adaptive implicit training systems. Given the current (and likely future) emphasis on Stabilization, Security, Transition, and Reconstruction Operations (SSTRO) and other socioculturally situated operations, intuition research is being conducted and developed for psychosocial skills training. In particular, the Virtual Observation Platform, a simulation-based trainer in development under PercepTS, could act as a test site for implicit learning techniques to enhance intuitive decision making. This simulation-based training approach offers opportunities for practice in sensemaking activities, via anomaly detection among patterns of human behavior. Repeated opportunities for practice can accelerate the development of expertise, wherein the sensemaking process of informed observation, analysis, and action becomes intuitive. Development of intuitive sensemaking will provide learners with the ability to solve ill-defined problems and make sound, complex decisions in uncertain, socially complicated operating environments.

\section{Additional On-going and Future Work}

Future work in this area might explore the use of adaptive training interventions, including neurophysiologically informed adaptive instructional systems currently being investigated by other researchers. For example, under DARPA's Warfighter Intuition 
effort, using a high-density EEG and post hoc analyses, researchers identified medial orbital frontal electrical responses that seem to correlate with presence of intuition (Luu et al., 2010). The cumbersome technology and time-consuming analyses prohibit this technology from being deployed in the near term; however, it is reasonable to believe that a neurophysiologically informed adaptive training system could support intuition instruction.

In Spring of 2013, work will begin on an ONR-funded program to address four areas of a Basic Research Challenge: "Enhancing Intuitive Decision Making Through Implicit Learning." A world-class team of researchers will combine the talents of the groundbreaking cognitive and neuroscience university laboratories at Northwestern University (NWU), Massachusetts Institute of Technology (MIT), and University of California, Los Angeles (UCLA) with teams of human systems modeling and simulation scientists from Charles River Associates, and Defense Group Inc., all led by MESH Solutions/DSCI to contribute to the Intuitive Sensemaking Interactive Simulation (ISIS) program. This unique team will collaborate to research, develop models, make recommendations, and test advanced instructional strategies for use in adaptive implicit scenario/simulation based training system.

The phased approach of the program will include parallel Neuroimaging experiments conducted at the IMHRO Staglin Center for Cognitive Neuroscience at UCLA and the Center for Translational Imaging at NWU to investigate multiple brain processes in order to gain greater understanding of intuitive decision making. Computational models will be developed and used for both data analysis and to inform recommendations for instructional strategies. Results in the form of modeling predictions and training recommendations will be used to drive simulation-based training experimentation and to explore the training effectiveness, and validation of, the resulting guidelines and strategies.

Current and future work will need to articulate and refine possible training strategies that could be triggered or enhanced by neurophysiological inputs and to recommend adaptive training strategies that a developer could implement into an intuition intelligent tutor, once the corresponding sensing technologies reach sufficient maturity. The overarching goal of this work is to advance capabilities for enhancing the intuitive decision-making skills of military personnel by investigating a range of intuition training approaches, situated in the context of urban sociocultural sensemaking.

Acknowledgement. This work was supported, in part, by the Office of Naval Research project N00014-11-C-0193, Perceptual Training Systems and Tools (PercepTS). The views and conclusions contained in this document are those of the authors and should not be interpreted as representing the official policies, either expressed or implied, of the Department of Defense or Office of Naval Research. The U.S. Government is authorized to reproduce and distribute reprints for Government purposes notwithstanding any copyright notation hereon. 


\section{References}

1. Agor, W.H. (ed.): Intuition in Organizations: Leading and Managing Productively. Sage Publications, Newbury Park (1989)

2. Betsch, T.: Intuition in Judgment and Decision Making. Taylor and Francis Publishers, New York (2008)

3. Claxton, G.: The anatomy of intuition. In: Atkinson, T., Claxton, G. (eds.) TheIntuitive Practitioner. Open University Press, Buckingham (2000)

4. Dane, E., Pratt, M.: Exploring intuition and its role in managerial decision making. Academy of Management Review 32(1), 33-64 (2007)

5. Dervin, B.: An overview of sense-making research: Concepts, methods and results. Paper Presented at the Annual Meeting of the International Communication Association, Dallas, TX (1983)

6. Ericsson, K.A., Lehmann, A.C.: Expert and exceptional performance: Evidence of maximal adaptation to task constraints. Annual Review of Psychology 47, 273-305 (1996)

7. Harper, S.C.: Intuition: What separates executives from managers. In: Agor, W.H. (ed.) Intuition in Organizations, pp. 111-124. Sage Publications, Newbury Park (1989)

8. Hassin, R.R., Uleman, J.S., Bargh, J.A. (eds.): The New Unconscious. Oxford University Press, Oxford (2005)

9. Hodgkinson, G.P.: Intuition in Organizational Decision making. Keynote address to the IST Workshop, February 4-5. University of Central Florida (2009)

10. Hogarth, R.M.: Educating Intuition. University of Chicago Press, Chicago (2001)

11. Klein, G.: Sources of Power: How People Make Decisions. MIT Press, Cambridge (1998)

12. Klein, G., Moon, B., Hoffman, R.: Making sense of sensemaking 1: Alternative perspectives. Intelligent Systems 21(4) (2006a)

13. Klein, G., Moon, B., Hoffman, R.F.: Making sense of sensemaking Ii: a macrocognitive model. IEEE Intelligent Systems 21(5), 88-92 (2006b)

14. Lowenstein, R.: When Genius Failed: The Rise and Fall of Long-Term Capital Management. Random House Trade, New York (2000)

15. Luu, P., Geyer, A., Fidopiastis, C., Campbell, G., Wheeler, T., et al.: Reentrant Processing in Intuitive Perception. PLoS One 5(3), e9523 (2010)

16. Moore, D.T.: Sensemaking: A Structure for an Intelligence Revolution. Clift Series on the Intelligence Profession. National Defense Intelligence College (2011)

17. Reber, P.J.: Cognitive neuroscience of declarative and non-declarative memory (2008)

18. Guadagnoli, M., de Belle, S., Etnyre, B., Polk, T., Benjamin, A. (eds.): Parallels in Learning and Memory, 113-123

19. Salas, E., Rosen, M.A., Diaz Granados, D.: Expertise-Based Intuition and Decision Making in Organizations. Journal of Management, 1-31 (2009)

20. Schatz, S., Wray, R., Folsom-Kovarik, J.T., Nicholson, D.: Adaptive Perceptual Training in a Virtual Environment. Paper presented at the Interservice/Industry Training, Simulation, and Education Conference (I/ITSEC), Orlando, Florida, December 3-6 (2012a)

21. Schatz, S., Nicholson, D.: Perceptual Training for Cross-Cultural Decision Making. In: Nicholson, D.M. (ed.) Advances for Design in Cross-Cultural Activities Part I, pp. 3-12. CRC Press, Boca Raton (2012)

22. Schatz, S., Folsom-Kovarik, J.T., Barlett, K., Wray, R., Solina, D.: Archetypal Patterns of Life for Military Training Simulations. Paper presented at the Interservice/Industry Training, Simulation, and Education Conference (I/ITSEC), Orlando, Florida, December 3-6 (2012) 
23. Simons, D., Chabris, C.: The Trouble with Intuition. The Chronicle of Higher Education (2010)

24. Sonenshein, S.: The role of construction, intuition, and justification in responding to ethical issues at work: The sensemaking-intuition model. Academy of Management Review 32(4), 1022-1040 (2007)

25. Thurlow, A., Mills, J.: Change, talk and sensemaking. Journal of Organizational Change Management 22(5), 459-579 (2009)

26. Weick, K.E.: The Social Psychology of Organizing, 2nd edn. McGraw-Hill, New York (1979)

27. Weick, K.: The Collapse of Sensemaking in Organizations: The Mann Gulch Disaster. Administrative Science Quarterly (38), 628-652 (1993) 